\title{
¿Anglosajón o romano?
}

\section{KATHERINe MilLeR \\ Directora de Asuntos Culturales Biblioteca "Florentino Idoate, S.J."}

\section{Oppe wenst Pu Pte on nigǔ menniscǔ $m$ ge auht $f$ str lices bion buton Hwearfunga?}

[Or did you think that any constant thing except changefulness could be in any man? Traducción propia] [ $¿ 0$ talvez pensabas que cualquier asunto constante, salvo una naturaleza cambiadiza pudiera encontrarse en el hombre? Traducción propia]

Selección de De Consolatione Philosophiae de Boecio en la traducción al anglosajón por el rey Alfred de los Ingleses, siglo IX

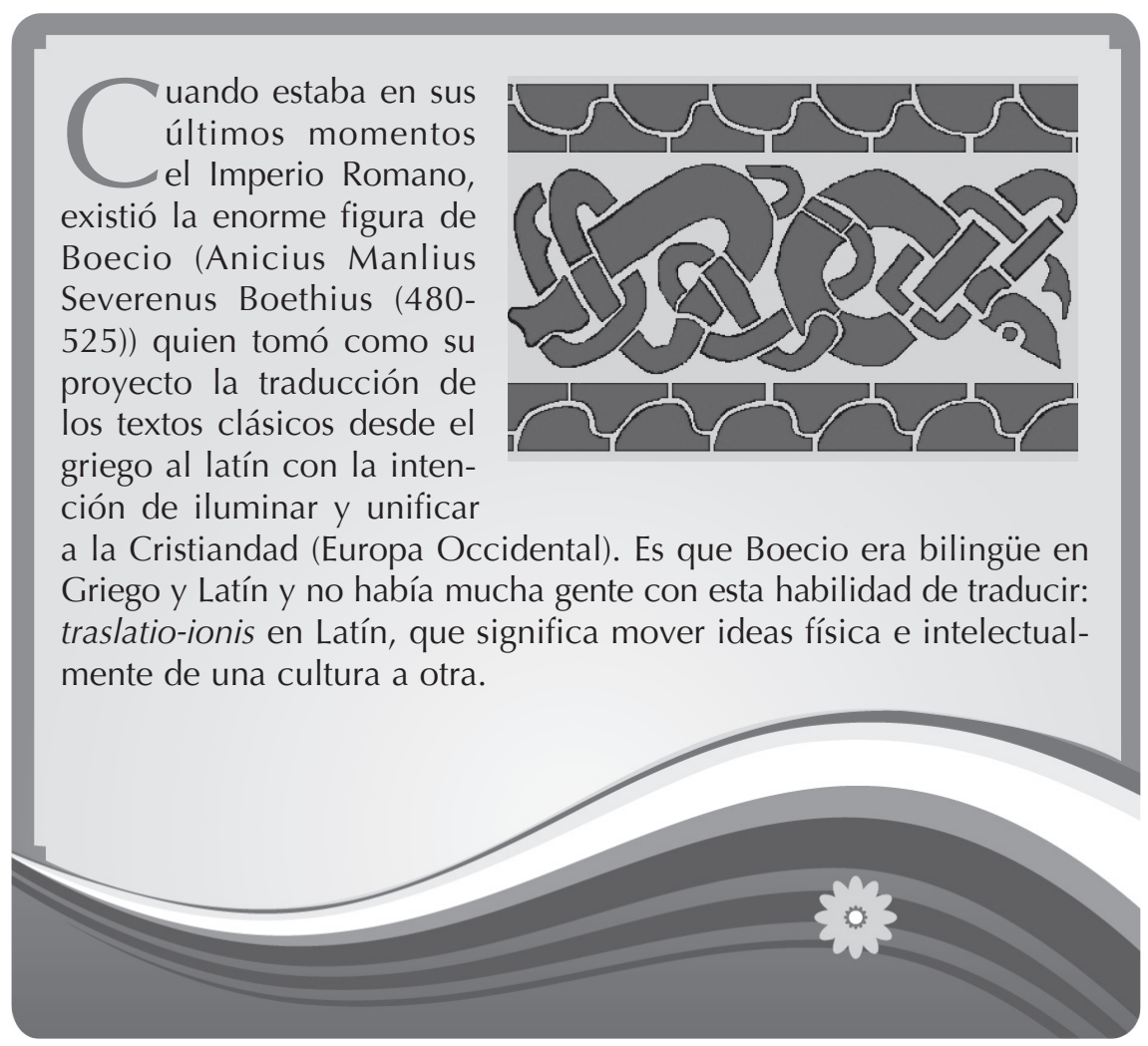


La Cristiandad de los tiempos de Boecio-lo que iba ser Europa Occidental - estaba experimentando un proceso de unificación que no era política. No habían estados ni naciones: estas regiones donde estaban creciendo los idiomas vernáculos (los dialectos de Inglés, Francés, Alemán, Español) no eran países, solamente lugares y regiones o pequeños reinos eternamente en guerras.

Desde el siglo VI, tiempos de Boecio, San Benito de Nursia (480 - 527) encabezó un movimiento para la unificación de Cristiandad. Esta reforma estaba particularmente en las manos de gente de la gran corporación transnacional y supranacional, la Iglesia Católica en Roma. En la iglesia habían brillantes personajes como Alcuino de York (730-804), un abad de un monasterio del orden monástico Benedictino en el norte de Inglaterra, en York.

Carlomagno convenció a Alcuino trasladarse a su corte para servir como Ministro de Educación y comenzar una reforma educacional para erradicar el analfabetismo de su reino y fundar escuelas para los niños (incluyendo puellae, niñas). Es que los monjes benedictinos entre los anglosajones eran los más eruditos de todo Cristiandad [Europa Occidental] en el siglo IX.

Simultáneamente con la vida de San Benito de Nursia, durante el siglo VI, Boecio estaba traduciendo la Lógica y otras obras de Aristóteles y los griegos al latín, en Verona. Monjes benedictinos, misioneros Anglosajones, copiaban su obra De Consolatione Philosophiae y lo llevaban al reino de Wessex en el sur de Inglaterra, donde Londinium (Londres) en la isla británica de Inglaterra y lo pusieron en las manos del rey Alfred (849-899). Este libro era la obra maestra de Europa Occidental por todo el período medieval. Alfred, un líder militar de Inglaterra, conformó su ejército y luchó contra las invasiones de los vikingos e intentaba, a la misma vez, unificar su reino. Este mismo Alfred tradujo la obra de Boecio del Latín a su dialecto de anglosajón, precursor del idioma Inglés en el siglo IX, para su pueblo. Eso lo hizo por considerarla de suma importancia para la construcción de su nación.

Inglaterra, la isla pequeña en el Mar del Norte, compuesto de cinco reinos que iba evolucionar y llegar a ser Inglaterra, había sido devastada por guerras desde que el ejército romano fue retirado en 410 E.C. Los vikingos de Noruega y Dinamarca estaban saqueando sus monasterios, masacrando no solamente los monjes, pero la población civil también. Los cinco reinos de la isla estaban en constante y violenta polarización bélica. Además, los vikingos daneses ocuparon militarmente una región grande en medio de la isla de los anglosajones y cobraban la renta, una extorsión extraída contra la amenaza de atacar a los 
anglosajones (Ingleses) y masacrar a sus poblaciones. El dinero de la extorción se Ilamaban Danegeld, literalmente, dinero para los daneses.

Alfred no era solamente un líder militar. Deseaba conformar un sistema de educación en la misma manera que su compatriota, Alcuino de York, había hecho para la corte de Carlomagno y educar a su pueblo además de defenderlos militarmente contra los invasores y los pleitos entre los cinco reinos de la isla. La unificación, defensa militar y educación de su pueblo eran sus metas declaradas.

Como vimos, durante el siglo IX, contemporáneo con Carlomagno, Alfred, en Londres, recibió de las manos de los misioneros muchos textos traídos desde Roma en la Península Itálica. Entre ellos era el texto de Boecio, De Consolatione Philosophiae. Alfred lo tradujo del latín al anglosajón del siglo IX (los manuscritos son del año ca. 880) para educar y, así, unificar a su gente. Su metodología era de "mover" (traslatio) las ideas de Boecio, el romano, al mundo de los anglosajones para su pueblo con la intención de elevar a sus mentes y salvarlos de su ignorancia y violencia. Era por eso que Alfred tradujo esta obra y otras al anglosajón.

En los tiempos de Boecio (siglo VI de la Era Común) y Alfred (siglo IX E.C.), la actividad representada por el término en Latín, traslatio ("translation" en Inglés; "traducción" en Español) no significaba la misma actividad que nosotros entendemos por este término. Literalmente, traslatio significaba mover una idea o un imperio - muchos asuntos - de un lugar a otro, agregando, para alcanzar su mayor recepción, tintes culturales, ideas indígenas a la cultura del nuevo idioma, conceptos de nación distinta de los del idioma original. Era una actividad de traducción cultural, más que nada. Nada de traducción rigurosa cercana al texto original que es característica de la actividad de traducción hoy.

Veamos, entonces, esta Consolación de la filosofía en anglosajón, traducido del Latín del siglo VI de Boecio por Alfred, el rey de Inglaterra, su líder militar quien tradujo este libro en medio de sus guerras, con el propósito de presentar a su pueblo un mito energizante. Eso lo hizo contando, en su traducción de la Consolación de la filosofía, en que un buen hombre, muy exitoso en este mundo cae, injustamente, y pierde todo, queda en prisión enfrentando la ejecución injustamente: pero es salvado por la personificación de la mente (Mod en anglosajón) quien simboliza la educación y entendimiento. Es la educación para su pueblo que, para Alfred, traduciendo la cultura Romano a la cultura anglosajón, es la inspiración hacia la paz y unificación de su nación porque la educación trae el entendimiento y la tranquilidad a 
la mente, como declaró Alfred por escrito.

La traducción al anglosajón de la obra De Consolatione Philosophiae de Boecio solamente se parece en parte al libro original en latín. Donde Boecio vio al universo en una manera platónica como una serie de círculos concéntricos proyectados de la mente de Dios, Alfred luchaba con el dualismo de la idea y la materia, en su intentotalvez sin éxito-de forjar una unidad, al estilo de Boecio, de los dos en la Inglaterra de los anglosajones.

Mi intención aquí es de tratar la traducción de Alfred como una obra sui generis escrita por un pueblo con una percepción del mundo que resistió, en muchas maneras, la concepción del orden romano. La diferencia entre las dos conciencias culturales se puede ver, durante el mismo siglo VIII, por ejemplo, en las iluminaciones fantásticas del Libro de Kells de los monjes célticos, en contraste marcada con las ilustraciones del Evangelio de Lindisfarne, copiado en Inglaterra después del Sínodo de Whitby, que decidió definitivamente a favor de la liturgia de Roma, dejando la liturgia de los celtas.

En fin, "germánico", "celta" y "anglosajón" son palabras de descripción del mundo, un Weltanschauung que sugiere sentimientos emocionales y filosóficos de los anglosajones, distintos de lo que nos comunica la palabra "romano". Es una visión del mundo anglosajón con sus intimaciones de fatum [Wyrd en anglosajón], exilio, muthabilidad, desesperación y melancolía con que Alfred comienza su traducción de Boecio: estos rasgos de conciencia cultural son los que marca la obra completa. Los esfuerzos de Alfred por subsumir bajo un idealismo al estilo de Roma, una visión anglosajón de su cultura, una visión esencialmente materialista y caótico en medio de las guerras, invasiones y divisiones del siglo IX en lo que iba ser Inglaterra, que presenta la tensión central en su trabajo de traducción de esta gran obra de Roma en latín de Boecio al inglés del siglo IX, al dialecto que hablaba la gente cerca de Londres: el anglosajón.

Pero hay pensadores e investigadores quienes han analizado el texto de Alfred como una expresión de existencialismo cristiano. Tal es el caso de F. Anne Payne en su publicación King Alfred and Boetius: an Analysis of the Old English Version of the Consolation of Philosophy (1968). La interpretación de Payne, escribiendo un análisis en el siglo XX de este texto del siglo IX, impone un tinte existencialista al texto de Alfred: 
What we encounter as we read the Old English text... is a Christian Existentialist preoccupied with the readjustment of every utterance of a Stoical Idealist, instead of with the forging of statements to express his own ideas. [Payne, p. 143].

[Lo que encontramos cuando leemos el texto del antiguo inglés es un existencialista cristiano preocupado con el reajuste de cada pronunciamiento del idealista estoico, en lugar de forjar pronunciamientos de sus propias ideas.] (Traducción propia)

Cuando Payne dice "Christian Existentialist", parece que ella quiere decir que el diálogo entre la Mente [Mod] y Sabiduría [Wisdom] se lleva acabo en un mundo en que falta el orden, y falta también un sentido de la eternidad omnisciente de Dios. El mundo que Payne encuentra en el texto anglosajón de Boecio, traducido por Alfred, es un mundo en que se percibe un "sense of the absurd disparity between the strength of man and the naked strength of Wyrd ". (Payne, p. 10). [El concepto Wyrd, en anglosajón, significa un concepto en que el destino, ya predeterminado, es inevitable, sin sentido e irreversible]. Pero Wyrd, para Alfred, como veremos, no es sinónimo con una visión existencialista, aunque sea Christiana. Intentaré comprobar que existencialismo no es una explicación aceptable si se lee el texto de Alfred, aunque superficialmente.

La interpretación de Payne puede ser resumido en el contraste que ella encuentra en las palabras dominantes utilizadas por los dos autores: "Being for Boethius, Be- coming for Alfred" (Payne, p. 140). ["Ser para Boecio, llegando a ser para Alfred." (Traducción propia).]

Los desacuerdos que deseo expresar con la interpretación que ofrece Payne están centrados particularmente en sus discusiones del orden y eternidad. Payne, en mi opinión, ha capturado, no obstante tan admirablemente, solamente la mitad de la visión de Alfred. Decir que una de las alteraciones más importantes de Alfred en su traducción del texto de Boecio es su sustitución de una teoría que es basado en la idea de la libertad, por una idea que es basado en el concepto de orden es de ignorar porciones significantes de la obra de Alfred. No creo que es posible decir, como declara Payne, que Alfred nunca utiliza el mundo natural como un símbolo de orden (p. 37); que para Alfred solamente existe el tiempo presente y actual (p. 138) y nunca una visión de la eternidad como un estado de ser en contraste con la eternidad (p. 56).

No, Alfred describe el orden 
natural como la encarnación o personificación del cambio, igual como de una visión cíclica y de orden. Estoy de acuerdo en que, durante muchas páginas del texto anglosajón, parece ser que existe solamente la lucha en esta vida presente aquí en la tierra. Pero, entonces, Alfred incluye muchas imágenes de la eternidad como un estado más allá de esta vida, y solamente por equivocación sería posible argumentar, como lo hace Payne, que Alfred no presenta la visión de un reino ordenado y posible encima de las tormentas de esta tierra, igual como la eternidad de la segunda muerte. La imposición de ideas como existencialismo sobre una obra del siglo IX no es, al fin y al cabo, exitosa.

Para demostrar como Alfred presenta visiones del idealismo y materialismo, el uso de los bienes de este mundo y un concepto de orden, examinamos su texto en el original. Se ha acusado a Alfred de crear un texto que es solamente una mezcla de Boecio y Alfred mismo. Por cierto, el texto anglosajón no es Boecio en traducción como se entiende el proceso en el siglo XXI. Alfred mismo declara a sus lectores que, algunas veces él tradujo sentido por sentido, algunas veces, palabra por palabra. Es traducción en el sentido de traslatio en que se está superponiendo unas nociones sobre otras: algo así como intentar hacer que un triangulo sea congruente con un círculo. El universo de Boecio, el romano del siglo $\mathrm{VI}$, es una serie de círculos concéntricos emanando desde la mente de Dios y esta visión, sencillamente, no corresponde muy bien con la realidad nacional de Inglaterra en el siglo IX, recuperando y cayéndose otra vez en guerras e invasiones de los vikingos de Dinamarca.

Pero Alfred hace un buen intento y termina, en su texto, con Dios manejando los asuntos, en una manera triunfal, desde la eternidad y el hombre intentando subir a Dios por medio de la sabiduría y la razón. No importa que algunas piezas de realidad no caben en el marco.

La obra, entonces, comienza, después de una historia corta de la vida de Boecio y su ejecución ordenado por Teodorico el Ostrogodo, en Ravena. Alfred comienza, en el estilo anglosajón, con una descripción de la angustia del hombre sabio:

Pa hit gelomp P se arwyrða wæs on swa micele nearnesse becom ... unrot, 7 ormod ...

[La versión en anglosajón es de W.J. Sedgefield, ed (Oxford, 1899. King Alred's Old English Version of Boecio De Consolatione Philosophiae, p. 7. En adelante, Sedgefield.] 
[Then it happened that the wise man became very anxious...dejected and despairing ...(traducción propia).]

[Entonces, pasó que el hombre sabio se sentió ansioso ...deprimido en desesperación .... (Traducción propia).]

La voz de Heofencund Wisdom [sabiduría que proviene del cielo] pregunta si la figura central, Mod [la mente del protagonista] se ha olvidado sus "armas" que recibió como líder militar y sus libros, ambos para luchar contra el mundo. Aquí, Heofencund Wisdom expresa una suerte de exorcismo-una declaración contra los espíritus del mal:

GewitaP un awirgede worulsorga of mines Pegnes mode, for $\bar{\alpha} \bar{\alpha}$ ge sind Pa mastan, sceaPan. LataP hine eft hwearfan to minŭ, larǔ [Sedgefield, p. 8].

[Go away now accursed worldsorrow from my servant's mind, for you are the greatest injury. Let him afterwards return to my teachings. (Traducción propia).]

[Váyase ahora, tristeza del mundo damnificado, de la mente de mi seguidor, porque usted es la injuria más grande. Después él debe regresar a mis enseñanzas. (Traducción propia).]

Wisdom limpia las lágrimas de los ojos de Mod, su mente y lamenta la discordia de Mod consigo mismo. Inmediatamente aparece una meditación-que no es una visión apocalíptica como el famoso
Sermo Lupi (homilía sobre el fin del mundo). No, es un bosquejo de la forma del mundo anglosajón donde el hombre cae en la desesperación como un exilio en la oscuridad sin la luz celestial de la Razón:

Pa ongan se Wisdom hreowsian for Pas Modes tyderness, 7 ongan Pa giddian 7 Pus cwæeð: Eala on hu grundeasum seað P mod drigð

Pŏn hit bestryrmað Pisse woruld ungePwarnessa. Gif hit Pŏn forget his ahgen leoht, $P$ is ece gefea, drigð on Pa fredan Pistro, $P$ sind woruldsorga, swa swa dis Mod nu deð, nu deð, nu hit haught elles nat butan gnornunga ... Sittað manfulle on heahsetlu, 7 halige under heora fotu Pyrcað; sticiað gehidde beorhte craeftas, 7 Pa unrihtwisan taclað Pa rihtwisan. Naught ne deregað топnи mane aPas, ne $P$ lease lot Pe beoð mid Pa wrencu bewrigen. ForPa went nu fulneah call moncyn on tweonunga, gif seo wyrd swa hweorfan mot on yfelra manna gewill, 7 Pu [God/ Dios] heore nelt stiran. [Sedgefield, p. 9-10] 
[Then began Wisdom to sorrow for Mind's frailty and began to sing and spoke in this way: Alas! How the Mind rushes into a bottomless pit when this world's discord agitates it. If it forgets its own light, that is, eternal joy, and rushes into the outer darkness, that is, the sorrow of the world, as this Mind now does, there is nothing else except lamentation ... wicked men sit on the throne and holy men are trampled under their feet; bright skills lie hidden and the unrighteous deride the righteous. Wicked oaths do not injure men at all, nor the false lots which are concealed with fraud. Therefore all mankind goes in doubt if Wyrd may change according to the will of evil men, and You [God] will not control her.]

\section{[Traducción propia]}

[En este momento Wisdom comenzó [sentirse] triste a causa de la fragilidad de la Mente y comenzó a cantar y habló en esta manera: ¡Hélas! como se apura la Mente a caer en un pozo sin fondo cuando la discordia de este mundo la agita. Si se olvida su propia luz, es decir, la alegría eterna, y corre hacia la oscuridad, es decir, la tristeza del mundo, como lo hace la Mente ahora, no hay nada salvo lamentación. [...] Hombres malvados están en el trono y hombres santos están pisoteados debajo de sus pies. Hhhabilidades brillantes están escondidas y los malintencionados desprecian los bienintencionados. Juramentos malos no causan injuria a los hombres, ni los apuestos fraudulentos que están escondidos debajo el fraude. Por lo tanto, toda la humanidad anda en duda si es el caso que Wyrd pueda cambiar según la voluntad de hombres males y Tu [Dios] no la controla.] (Traducción propia)

Mod es presentado como una especie de hijo pródigo que ha vagabundeado fuera de las tierras de su padre y es ya un exiliado. No fue víctima de seducción por diablos o mala compañía, pero, como Wisdom lo explica: "Ne gebrohte Pe eac nan oPer man on Pa gedwolan butan Pe sylfum Purh Pine agene gemeleste." [No other man brought you into this error but you yourself through your own negligence] [Ningún otro hombre le trajo a estos errores sino tu mismo por su propia negligencia. (Traducción propia)] Así es que Mod sufre ormodnesse, una agitación de la mente que lo ha causado sentirse no alineado con el mundo: "ForPamPu scedest Pu wraecca ware ..." [Therefore you said you were an exile] [Por lo tanto dijiste que era un exiliado].

Wisdom ha entrado la mente $y$ el diálogo se lleva acabo en un espacio interno, en su min murniende 
mod [my agitated mind] [Mi mente en estado de agitación]. El libro procede como una confesional y catequesis: frecuentemente Wisdom aparece como madre adoptiva, formula una pregunta $y$, después, él lo contesta él mismo. Nota que Wisdom es en el género masculino, aún cuando es presentado como "madre adoptiva".
La discusión ha comenzado con Boecio estirado en el suelo de la carcel envuelto en desesperación y tristeza. El mundo visto desde su punto de vista-y lo de Mod-es principalmente un lugar de lamentación, cambio, pozos sin fondo, oscuridad, frío, exilio y maldad. Wisdom reconoce esta melancolía germánica e intenta, paulatinamente, hacer un bosquejo de explicación:

Hwat, Pe ealle gesceafta heorsumiað, 7 Pa gesetnesse Pinra beboda healdað, butan men anu, se Pe oferheorð. [Sedgefield, p. 14]

[Lo, the whole creation obeys and holds to your [God's] commands except men alone, who disobey. (Traducción propia)]

[Considera como la creación entera obedece y sigue los mandamientos de Dios, salvo hombres, quienes desobedecen.]

Mod queda cubierto con neblinas como cuando el sol es cubierto con nubes, como el mar turbulento, como un riachuelo que ha sido dividido por una piedra grande. $\mathrm{Y}$, por eso, no está en la estación propia par sembrar Razón y Entendimiento: es untiidlice [untimely] [momento inoportuno], como cuando uno siembra en el sol caliente de agosto.
Wisdom, aparentemente, tiene los asuntos bajo control y el hombre solamente tiene que voltearse hacia heofencunde Wisdom para ver $y$ entender como funciona el mundo. Pero, de allí hay un desliz hacia atrás en el caos del tiempo actual con Wisdom mismo, de repente, gritando en frustración:

Eala, hu yfele me doð manige woruldmenn mid Pa $P$ ic ne mot wealdan minra agenra Peawa. Se heofen mot brengnan leohte dagas 7 eft $P$ leoht mid Peostrum behelian; $P$ gear mot brengnan blostman 7 ealle gesceaftga motan hiroa gewunan 7 heora willan bewitigan butan me anum. Ic ana com benuman minra Peawa 7 eom getogan to fremdu Peawu Purh Pa ungefylledan gitsunge woruldmona. Purh Pa gidsunga hi me habbað benumen mines naman Pe ic mid rihte habban sceoldan. [Sedgefield, p. 17]

[Alas, in what evil ways many worldly men act toward me that I 
may not govern my own servants. Heaven may bring light days, and afterwards obscure them with darkness; the year might bring forth blossoms, and the same year take them away. The sea might enjoy calm waves and all creation might keep their customs except me alone. I alone am deprived of my servants and am doomed to foreign customs through the insatiable greed of worldly men. Through greed they have taken away my name which I ought by right to have. (Traducción propia]

[[Hélas, en tantos maneras de maldad muchos hombres mundanos se comportan hacia mi que yo no puedo gobernar mis propios seguidores. El cielo pueda traer días de luz, y después oscurecerlos con la noche; el año pueda traer flores y el mismo año las quita. El mar puede gozar de olas tranquilas y toda la creación pueda mantener sus costumbre salvo yo solo. Solamente a mi me quitan mis sirvientes y estoy predestinado a [vivir con] costumbres de extranjeros a causa de la avaricia insaciable de los hombres mundanos. Por medio de la avaricia, han quitado mi nombre que yo debo tener como derecho. (Traducción propia)]

En el texto de la obra entera, Alfred ciertamente se mueve hacia el orden, eternidad y el amor de la visión romana del mundo aquí en la tierra. Pero la poesía fuerte y oscura de los Anglosajones, de repente, irrumpe, alternando con la visión desde arriba de la tormenta, así como si fuera un patrón de razón y entendimiento que está siendo forsozamente, sin éxito, impuesto encima de lo que es mutable y opaco en el mundo anglosajón:

OPPe wenst PuPte on aenigu menniscu mage auht fastradlices bion buton hwearfunga? [...] Eala P nanwuht nis faste stondendes weorces a wuniende on worulde. [Sedgefield, p. 20]

[Or did you think that any constant thing except changefulness could be in any man. [...] alas that nothing fast-standing is ever in this world.

Traducción propia]

[¿O talvez pensaba que cualquier asunto constante salvo una naturaleza cambiadizo pudiera encontrarse en el hombre? (Traducción propia)] 
¿De que cantan los poetas, pregunta, si no de los cambios continuos de este mundo? El Orden y la Razón ceden a cambio por la inseguridad y Wyrd, pero, enseguida, de nuevo están empujado, formado otra vez en los patrones de orden.

El problema de Payne es que solamente capta y representa la angustia y lo absurdo del reino de Wyrd en la tierra y, por su conveniencia, no reporta las visiones de alternancia entre el cambio de regreso al orden eterno. Payne enfatiza la libertad y el mundo que existe en el mundo actual, incluyendo, en su análisis, nada de la eternidad que Alfred insiste en incluir. El oportunismo del análisis en que Payne muestra, en su libro, solamente el presente y lo que queda es, únicamente, el tiempo y las secuencias de la vida y acción. Ella comenta que Alfred, en su traducción de Boecio, presenta solamente

"a universe largely contingent on present time. [...] Alfred never proves anything, simply because, as long as a writer is concerned with the passage of time, he cannot draw pictures of an eternal idea. [...] he left himself nothing but uncertainty and a few assertions that he elected to make: that men have the nature to follow good, that they are allowed to repent" (pp. 138-9).

[Un universo que existe y depende solamente en el tiempo actual. [...] Alfred nunca comprueba nada, sencillamente porque, mientras que un escritor está preocupado con el pasaje de tiempo, no puede dibujar cuadros de una idea eterna. [...] no se deja nada mas que el incertidumbre y unos cuantos aseveraciones que él decidió hacer: que los hombres gozan de la naturaleza de seguir el bien, y que son permitidos a arrepentirse." (Traducción propia)]

Payne declara que Alfred sustituye la idea de libertad para la idea del orden; que nunca vincula el orden con Wyrd; ni emplea estados de tiempo y eternidad, uno en contraste con el otro.

Mi respuesta a estos pronunciamientos es que algunas veces así lo hace Alfred, y algunas veces, no lo hace así. Pero es importante no omitir ninguno de los dos actitudes para conformar (¿distorsionar?) un texto para que cuadre con un hipótesis. Examinamos unas selecciones en las que Alfred, por cierto, contrasta el tiempo actual, desordenado y en desesperación con el orden de la eternidad.

Gif Pu un getelest Pa hwila Pisses andweardan lifes 7 Pisses hwilendlican wið ðœes ungeeodan lifes hwile, hwat bið hit Ponne? [...] Tele nu Pon P 
ten Pusend geara, ge Peah Pu ma wille; wið Pece 7 Pat ungeendode lif; $P$ on ne finst $P u$ Pat naught angelices, forPan $P$ ten Pysebd gearam Peah hit lange Pince, ascortaP, 7 Pas oðres naefre ne cymð nan ende. ForPam hit nis no to metan ne P geendolice wið Pat ungeendodlice. [Sedgefield, p. 37-38]

[If you now consider the length of this transitory and present life and the length of the unending life, what is it then? Consider now ten thousand years, even more if you want, with the eternal and neverending life; then you will not find there anyting like it, because ten thousand years, though it seems long, will shorten, and the other will never come to an end. Therefore it is not to be compared, the ending with the never-ending.(Traducción propia)]

[Si usted considera, ahora, como es de larga esta vida actual y transitoria y como es la vida sin fin, ¿qué es, entonces? Considere ahora diez mil años, más si así lo desea, porque diez mil años, aunque parece largo, se vuelven más cortos y el otro nunca terminará. Por lo tanto, no se debe comparar lo que va a terminar con lo que nunca termina. (Traducción propia)]

Otra vez, Wisdom explica:

Forðon Pe we witon swiðe lytel ðæs Pe ær us wæs buton be gemynde 7 be geæscum, 7 get læsse Pæs ðe æft us bið. P an us is gewislice andweard P Pe Ponne bið; ac him is eall andweard, ge Pte ær wæs, ge Pte nu is, ge Pte æfter us bið; eal P is him andweard. [...] syle he biǒ ece, forPæm næfre sio tid næs P he nære, ne næfre ni wyrð.

[Sedgefield, p. 148]

[Therefore we know very little of that which was before us, except by memory and by enquiry; and still less of that which shall be after us, that alone is truly present to us which at the time is; but to him all is present, both what was before, and what now is and after us shall be; it is all present to him. [...] He is always eternal, for the time never was when he was not, nor ever will be. (Traducción propia)]

[Por lo tanto, sabemos muy poco de lo que existía antes de nosotros, salvo por medio de la memoria, o por investigación; y menos todavía de lo que será después de nosotros, y solamente sabemos de lo que es en verdad presente a nosotros en el tiempo actual; pero a él todo es presente, lo que existía antes, y lo que es y lo que después de noso- 
tros será; es todo presente a él. [...] Él es siempre eterno, por el tiempo nunca fue cuando él no existía, ni nunca será. (Traducción propia)]

Estas selecciones establecen la mitad de la respuesta de Alfred a la pregunta sobre las relaciones de hombre y eternidad (Dios), i.e., la existencia e importancia del anterior. La segunda porción de la pregunta, sobre la clase o categoría de la relación, es lo que no concuerda con el texto de Alfred. La aseveración de Payne que el texto de Alfred es, en esencia, existencialista no es posible. El hombre y la eternidad de Dios, sí tienen una relación en el texto anglosajón. No se puede negar el vínculo íntimo (causal) entre ellos que constituye la inmanencia de Dios en la visión de Boecio. Tampoco es el caso en la Consolación de la filosofía de Alfred.
Es claro que Alfred está luchando con una visión particularmente anglosajona del universo, buscando moverlo hacia la órbita del idealismo y orden presentada por Boecio. En el hecho de terminar su obra con la armonía eterna de las esferas, Alfred induce su lector a creer que ha cumplido con la confección transformada de la visión anglosajona. Pero, como veremos, eso lo logra solo parcialmente y no más.

Hay que ver su progreso en este proceso como una puente de suspensión, inestable, colgada entre este mundo y Dios, conectando los dos por yuxtaposición y alternación. Pero las imágenes más vívidas que Alfred presenta de este mundo son imágenes en que la razón es ausente:

Seite Pas lichoman is swiðe flionde 7 swiðe tedre 7 swiðe anlic eorðan ostmum. [Sedgefield, p. 172]

[The beauty of the body is very fleeting and very frail and very like The flowers of the earth. (Traducción propia)]

[La belleza del cuerpo es escurridiza y muy frágil y muy así como las flores de la tierra. (Traducción propia)]

De otro modo, Alfred nos enseña el mundo natural como emblema del orden también, no obstante la insistencia de Payne que nunca demuestra el mundo natural como un símbolo de orden (Payne, p. 37). Lo siguiente es uno de los himnos que Wisdom canta a Dios, quien reina sobre la creación con sabiduría: 
Pundorlic is P Pin gePeaht, P ठu hafst aeghPergedon: ge Pa gesceafta gemaescode betwux him, ge eac gemengde Pa drigan eorðn 7 Pa dcealdan under Pa cealdan watere 7 watan, P Pat hnesce 7 flowende water habe flor on Pare fastan eorðan; foPaPe hit ne mag on him selfu gestandan. Ac seo cor e hit helt 7 be sumu daele swilgð, 7 for Pa sype heo bið geleht $P$ hio grewð 7 blewð 7 westmas bringð; forPa gif $P$ wat hi ne geðwande, Pon drugode hio wurde todrifen mid Pam winde swa swa dust oððe ace. [Sedgefield, p. 79]

[Wonderful is your plan, that you have done both things; you have both bound the creatures between themselves and also mixed them, the dry

And cold earth under the cold and wet water, that the soft and flowing water has a floor on the firm earth, because it cannot stand by itself. But the earth holds it, and in some measure swallows it and by moistening it is lightened that it grows and blossoms and brings forth fruit; for if the water did not wet it, then it would dry out and be driven by the wind as dust or ashes. (Traducción propia)]

[Maravilloso es su plan, que usted ha hecho ambas cosas; usted ha amarrado las criaturas entre ellos mismos y también las ha mezclado, la tierra seca y fria debajo del agua frió y mojado, en tal manera que el agua goza de un suelo de tierra firme, porque no puede pararse por si solo. Pero la tierra lo mantiene abrazado, y, de alguna medida, lo traga y así vuelve más lijera para que crezcan y florezcan y produce fruta; pero si el agua no lo mojara, entonces sería seco y empujado por el viento como polvo o cenizas. (Traducción propia)]

Alfred es conciente de la naturaleza errada y transitoria del mundo pero, a la misma vez, asume el orden racional de la creación de Dios que permanece en la eternidad. Y no obstante este dualismo, continúa su lucha hacia la racionalidad, cristiandad y la Iglesia Romana.

Referente a mi pronunciamiento sobre el materialismo arriba, pienso que la visión anglosajón del siglo
IX es una visión esencialmente materialista en que lo más alto que se puede alcanzar es el honor, elogio y gloria en las batallas de este tierra. La victoria en batalla puede ser permitido por Dryhten [Dios en su aspecto anglosajón como líder militar en batalla] y talvez escucharemos oraciones a Él. Eso es parecido, en gran medida, a la percepción de ese mundo expresado por Mod [la mente del protagonista]. Aún si Wisdom reconoce de vez en cuando, en 
sus descripciones de la mente del hombre o la pérdida de sus sirvientes, etc., la visión comprensiva del universo creado en las palabras de
Wisdom es un universo bajo el control de Dios. Visiones más adelante expresan un malestar y temores de caos que amenaza.

An sceppend is buton tweon, 7 se is eac 7 wealdend heofones 7 eorðan 7 ealra gesceafta, gesewenlice 7 eac ungescwenlicar; $P$ is God celmihtig [...] Se ilca gesette unawendendlice sido 7 Peawas 7 eac gecyndelice sibbe eallu gesceaftu, $P a P a$ he wolde, 7 swa swa he wolde; Pa nu standan to worulde. Dara unstillena gesceafta styring ne mag no weorðan gestilled, ne eac onwend of Pam ryne 7 of Pare endebyrdnesse Pe him geset is; ac se anwealda hafð calle his gesceafta swa mid his bridle befangene 7 getogene 7 gemanode $P$ hi nanPer ne gestillan ne moton, ne eac swið or styrian Pon he him Pat geru his wealdle deres to forlat. [...] P hie ne moton toslupan .... [Sedgefield, p. 48]

[There is one creator without doubt and he is also the ruler of heaven and earth and of all Creation, visible and invisible; that is God almighty. [...] The same has appointed unchangeable customs and habits and also natural kinship to all of creation when he wants to, and as long as he wants to, which now stand in the world. The constantly moving creation may not become still, nor even turn from the course and from the order which is set for it; but the powerful one has caught hold of his creation as with a bridle and restrained and admonished that it may neither be still nor move, nor yet more than he allows with the free play of his reins. [...] So that it might not slip away.... . (Traducción propia)]

[Hay un creador sin duda y él es también el que reina en los cielos y en la tierra y en toda la creación, visible e invisible; este es el Dios todopoderoso. [...] Lo mismo ha decidido las costumbres y prácticas que no cambian y también un parentesco natural para toda la creación de lo que es ahora en el mundo cuando él desea hacerlo, y mientras él desea hacerlo. La creación en constante movimiento no puede quedar quieto, ni jamás desviar del camino y del orden que ha sido impuesto; pero él que es poderoso ha agarrado su creación así como por medio de una brida [lit., de un jinete con un caballo] y la ha restringido y la ha corregido para que ni puede quedar sin moverse, ni moverse, ni un poco más que lo que él desea permitir con la rienda suelta de sus riendas [lit., Dios como jinete]. [...] Así, no puede deslizarse.] 
Dios reina sin esfuerzo y todas las criaturas (menos el hombre y algunos ángeles) por su propia volun- tad, voltean sus naturalezas hacia la voluntad de Dios:

Ne mag nanne mon Pas twiogean Pte ealra gesceafta agnu willum God ricsað ofer hi, eaðmodlice hiora willan wendað to his willan. [...] Nis nan gesceaft Pe tiohhie $P$ hio scyle winnan wið hire scippendes willan gif hio hire cynd healdan wille. [...] Da smearcade he [Wisdom] 7 cwar: Wite geare ðar is $P$ hehste god, ðat hit eall swa mihtiglice macað 7 eall ðing gescop 7 callu swa gereclice racað 7 swa eðelice buton alcu geswince hit eall set. [Sedgefield, p. 97-98]

[No man can doubt that, by all the creatures' own wills, God rules over them. [....] There is no creature which attempts to struggle against its Creator's will if it would keep to its nature. [....] Then he [Wisdom] smiled and said: Know assuredly that that is the highest good, that it mightily made and shaped all things and extends so widely and so easily without any labor, establishes it all. (Traducción propia)]

[Ningún hombre puede dudar que, por las voluntades propias de las criaturas, Dios reina sobre ellos. [....] No hay ninguna criatura que intenta luchar contra la voluntad de su creador si desea mantener su naturaleza. [....] Entonces, él [Wisdom] sonrió y dijo: Toma por acertado que eso es el bien más alta, que con fuerza hizo y formaba todas las cosas y extiende tan ampliamente y tan fácilmente sin fuerza de trabajo, y lo establece todo. (Traducción propia)]

En su libro, Payne niega que Alfred declare que las criaturas actúen de esta manera por un amor común, sirviendo a Dios. No obs- tante, podemos ver que Alfred, en su traducción de Boecio, declara que hacen exactamente eso:

Peah habbað gemanlice Pa ane lufe P hi Peowian swilcu hlaforde [...] Nis $P$ nan wundor forPam hi ne mihton elles bion, gif hi ne diowedon hiora fruman. [Sedgefield, p. 136].

They have one love that they owe to such a lord. [...] it is no wonder therefore that they may not else have being, if they do not serve their lord. (Traducción propia)] 
[Aman con un solo amor que deben a tal señor. [...] no es de admirarse, por lo tanto, que, de ninguna otra manera, puede existir, si no sirven a su señor. (Traducción propia)]

Alfred ha construido imágenes en su prosa que serían familiares a su gente. Al final de su libro, sin embargo, explica que el hombre no deberá entender a la grandeza

de Dios en una manera corporal ni material, pero en una manera espiritual, porque Dios es sabiduría y la sabiduría es espiritual:

His micelnesse ne mag nan man ametan; nis $P$ deah no liculice to wenanne, ac gastlice, swa swa un wisdom is 7 rihtwisnes, forðcm he Pis is self. [Sedgefield, p. 148]

[No man can measure his greatness; although this is not to be understood bodily, but spiritually, as wisdom is and righteousness; because he is that himself. (Traducción propia)]

[Ningún hombre puede medir su grandeza, aunque su grandeza no deberá Ser entendido corporalmente, sino espiritualmente, como sabiduría y actuación correcta; porque estos son él mismo. (Traducción propia)]

Esta declaración parece darnos a entender que, aunque en tiempos más temprano, Dios pudiera haber sido explicado en términos de hhhabilidades físicas, ahora lo debemos entender como un espíritu que permanece dentro de los seres humanos sin esfuerzo, guiando la creación en una manera libre, con hhabilidad y no con fuerza bruta. En su metáfora en que Dios aparece como jinete, guiando su creación como su caballo [probablemente un caballo de guerrero], debemos de estar conscientes de que uno guía un caballo con hhabilidad y ligereza, no con fuerza bruta.

Pero, hay otras partes del texto de Boecio traducidos por Alfred en que nos da a entender que la conciencia de los anglosajones no deja de interpelar, y Alfred tiene que describir también una especie de Götterdämmerung, muy familiar a los anglosajones. En estas declaraciones, es, sorprendentemente, Wisdom, y no Mod, quien está hablando:

Ac Ponne aer Pe P gewealdleðer forlaet Para bridla Pe he ða gesceafta un mid gebridlode hæð: Pis seo wiðerweardnes Pe we aer ymbe sprceon: gif he Pa laet toslupan, Pon forlactað hi $P$ a sibbe ðe hi un healdað, 7 winð heora 
ele on oðer afret his agenu willan, 7 forlað heora geferradenne, 7 fordo ð calne ðysne middangeard, 7 weorðað him selfe to nauhte. [Sedgefield, p. 49]

[But whenever he lets go of the reins of the bridle with which he has now bridled the creation, that is, the contrary things [lit., matters which ought to be undercontrol, but which have slipped out of control] which we spoke of before; if he lets the reins slip, then they forsake the kinship which they now hold and struggle each with the other after his own will, and they forsake their companionship and destroy all of this middle earth and they become nothing. (Traducción propia)]

[Pero en cualquier tiempo que él [Dios] deja rienda suelta la brida con que él ahora ha tenido controlado la creación, las cosas contrarias [lit., asuntos que deben ser controlados pero ahora están indebidamente sin control] de los que hemos hablado, estos asuntos abandonan la relación de parentesco que ahora tienen, y se ponen a luchar uno contra otro según su propia voluntad y ellos abandonan su sociedad y destruyen toda esta tierra y vuelvan a ser nada. (Traducción propia)]

Ahora, como es Wisdom, y no Mod quien está hablando, estas palabras de profunda melancolía y caos al enfrentar la disolución del mundo creado, parecen representar un lapse, un desliz hacia el paganismo ya que no es heofecunde Wisdom [sabiduría celestial de los cielos]. No obstante, como vemos aquí, es posible que Dios todavía puede comportarse en la misma manera que Wyrd [el fatum, destino inflexible que permea el mundo anglosajón del siglo IX] si, por casualidad, Dios, siempre como jinete, deja caer las riendas.

Este sentimiento, yuxtapuesto con las selecciones citadas anteriormente, comprueba el argumento que yo deseaba plantear: que en la traducción - traslatio- cultural del mundo romano al mundo anglosajón, visiones germánicas, o sajonas, irrumpen; pero el progreso hacia el orden romano continua de todos modos. Estas partes oscuras y amenazantes (talvez representando la violencia de las guerras internas $y$ las masacres y saqueos de los vikingos) no están incorporados completamente en la filosofía tipo romana bajo construcción por Alfred porque es difícil asumirlos en el mundo anglosajón, tan real y presente en el mundo en que está traduciendo el Romano Boecio. O sea, son intrusiones vívidas de la realidad anglosajona no tan fácilmente abandonadas, talvez porque todavía las estaban viviendo diariamente. 
La meta que Alfred plantea para sí mismo es de reconciliar el tiempo y los desastres de este mundo anglosajón con la visión romana de orden emanando desde Dios en la eternidad.. Para intentar eso, tenía que tratar, también, la cuestión del uso de los bienes de este mundo. $\mathrm{Y}$ esta, precisamente, es uno de los temas centrales de su traducción de la Consolatione de Boecio. La riqueza, la dignidad y el poder, como son los bienes más altas de esta vida actual, deben ser sirvientes de Wisdom. De hecho, Alfred presenta a Wisdom explicando que ningún bien puede ser la ocasión de separarse de Dios:

Gif Pa good waron Pare soðan gesclð limu bioP hwathewegu todaed; swa swa monnes lichoman [...] Pu ongitst P Pa good ealle sint P ilce P gesald, 7 sio gesalð is dat hehste good, 7 P hehste good is God, 7 se God is semle on anum untodceled. [Sedgefield, p. 87]

[The goods are limbs of the true happiness, they are a little separated, as man's body. [...] You perceive that the good is all the same as happiness is, and happiness is the highest good and the highest good is God and God is always one unseparated.(Traducción propia)]

[Los bienes son apéndices de verdadera felicidad [lit., en el sentido de brazos y piernas de un cuerpo humano]. [...] Usted puede percibir que el bien es lo mismo que la felicidad y que la felicidad es el bien más alto y el bien más alto es Dios y Dios siempre es uno, no separado en partes. (Traducción propia)]

Wisdom, además, ha explicado anteriormente, que es posible que él pueda perder el control-de hecho, ya, en ocasiones lo ha perdido: este es el control sobre sus sirvientes [los bienes de este mundo]. De otro modo, Mod recibe elogios por haberlos despreciado aun mientras que él los había tenido en su posesión: nos da entender que los bienes del mundo vienen a la mente sola- mente para separar la mente de las cosas que debe amar mejor.

Los bienes de este mundo son cosas indiferentes y están dispuestos a servir a hombres de mal, como seguidores rebeldes representado como thegnes, lit.,compañeros de alta alcurnia en batalla. Uno deberá optar por las cosas del cielo:

Ac Pa heofencundan Ping ðe sint gecynde, næs Pæs eorðlican. Pas eorðlican wæstmas sin gesceopene netenum and lifene.

[Sedgefield, p. 30] 
[But heavenly things are natural to you, not these earthly things. These earthly fruits have been created solely for sustenance and for cattle. (Traducción propia)]

[Pero las cosas del cielo son naturales a ustedes, no estas cosas de la tierra. Estas frutas terrenales han sido creado [solamente] para sobrevivir y para [el consumo del] ganado. (Traducción propia)]

Alfred escribe que los hombres entiendan como si fuera un sueño y que esta vida actual es como una sombra. Los hombres, dice, alcanzan a Dios por medio de un proceso mental de encender la chispa o grano de verdad en sus mentes. Las cosas del mundo (woruldsalða) son el agregado de todos los bienes y poderes en la vida actual en esta tierra, y así, forman parte de Dios como ente immanente en la creación. A la misma vez, sin embargo, Alfred explica, como paradoja, que estos woruldscelða son también sirvientes de Wisdom, monedas indiferentes del mundo, emblemas de la muerte que se debe despreciar $y$, a la misma vez, imágenes del bien eterno. El hombre goza de la felicidad (es decir, entendimiento, memoria y voluntad) y debemos utilizar a la felicidad para gobernar las posesiones con templanza e humildad, evitando la avaricia y arrogancia.

El hombre verdaderamente sabio debe odiar a los bienes del mundo y buscar y gozar de los bienes de la mente:

For Pam simle se wisa mon eall his lif lat on gefean unonwendendlice 7 orsorg, Pon he forship agðer ge Pas eorðlican god ge eac Pa yflu, 7 hopað to Pa toweardu; P sint Pa ecan. [Sedgefield, p. 46]

[Likewise, therefore, the wise man leads his whole life in unending joy and security when he despises both earhtly good adn also earhthly evil and trusts in the future which is eternity. (Traducción propia)]

[De igual manera, por lo tanto, el hombre sabio pasa toda su vida en alegría sin fin y en seguridad cuando odia los bienes del mundo y también el mal de este mundo y confía su futuro en la eternidad. (Traducción propia)]

Al leer las palabras arriba, parece que Alfred está intentando rechazar los bienes del mundo en favor de sabiduría, aun sabiendo que a estos bienes uno tiene que utilizarlos en esta vida. Estas dos ideas se juntan en el Capítulo XVII donde, con astucia, los bienes del mundo 
aparecen como sub-divisiones de Wisdom cuando Alfred está considerando los reyes y sus deberes. Mod [la mente en diálogo] declara que no desea el poder terrenal. Aunque había perdido este poder, al principio del libro, de repente lo encontramos intentando entender cómo manejar el poder terrenal en una manera "sabia" ya que posee el poder, y necesita de los materiales apropiados para su oficio como rey. Alfred tradujo como "craft" (hhabilidad casi artesanal), las hhhabilidades de gobernar como líder militar y político de una nación.

Un rey no debe despreciar hhhabilidades en su oficio (craft), porque, de todas las cosas terrenales, solamente esta hhabilidad perdura:

Pa craftegan ne mag nafre his craft losigan, ne hine mon ne mag Pon eð on him geniman ðe mon mag Pa sunnan awendan of hiere sede. [Sedgefield, p. 46]

[For the skillful man may never lose his craft, nor can any man more easily take it from him than one can move the sun from her place. (Traducción propia)]

[Es que el hombre con hhabilidades y capacidades nunca perderá estos, ni puede cualquier hombre quitarlo estas hhabilidades mas que uno puede mover el sol de su lugar. (Traducción propia)]

Parece que Mod ha llegado a ser un rey durante el Capítulo XVII y recibió ordenes de comportarse como tal. Además, ningún hombre puede mostrar ninguna habilidad o ejercicio del poder, sin las herramientas y materiales necesarias para su oficio. Aquí declara que, en la condición de ser rey, el uso propio de los bienes del mundo y aquí está la síntesis de Alfred, la solución al problema de cómo estar en el mundo.
Uno desprecia los bienes del mundo como buenos en sí, pero los utiliza para el ejercicio del oficio. Y así declara Boecio también en la versión latina. Pero el bien propio al hombre, dice, es el gozo de los bienes de la mente- este es el oficio del alma encarcelado en el cuerpo, en el mundo, pero anhelando la chispa divina que lo lleva al cielo, que es la sabiduría (wisdom).

Este es una síntesis inestable e incómoda, porque uno debe tener la voluntad y el poder de ejercer su oficio:

Twa ding sindon ðe alces monnes ingeðonc to fundað, $P$ is ðonne willa 7 anwald. [Sedgefield, pp. 9-10] 
[Two things are needed for man's exercise of his abilities, and these are will and power. (Traducción propia)]

[El hombre necesita dos cosas para ejercer sus habilidades, y estas son la voluntad y el poder. (Traducción propia)]

Para la mayoría de los hombres, nada es jamás suficiente y caen en la avaricia. Así que, Alfred tiene que balancear sus recomendaciones entre una actitud de desprecio hacia los bienes del mundo como bienes en sí, y la necesidad de estas mismas herramientas para gobernar. A la vez, uno deberá despreciar los bienes de este mundo porque interfieren con la búsqueda de la sabiduría de Dios.

En eso, Alfred necesariamente confunde las categorías de lo ideal con lo real, o material. Sabía que, aun mientras está elogiando los gozos de la mente, que los hombresespecialmente los reyes y líderes militares, tenían que tratar con los asuntos de esta tierra. Las ideas no efectúan cambios en el mundo sensible. Se necesita las herramientas materiales para dar forma al caos de las guerras y el mal en una manera en que los sirvientes (bienes) puedan ser restaurado a Wisdom mismo.

Ahora, Mod (quien, en este caso parece ser Alfred mismo) desea ser guardado en la memoria de su pueblo para sus buenas obras. Pero la respuesta inmediata de Wisdom a la expresión de este pensamiento es un rechazo y castigo. Wisdom mantiene que el deseo para esta gloria y poder en esta vida actual y el anhelo para la fama de buenas obras son, en sí, productos del mal:

Eala, Mod, eala; an yfel is swiðe to anscunianne [...] P is Pon wilnung leases gilpes / unryhtes anwealdes / ungemetlices hlisan godra weorca ofer eall folc. [...] Is P Ponne fordyslic gesine P ge wijnnað cowre woruld to ðо ðқt ge wilniað cowerne hlisan ungemetlice tobrcedanne ofer swelene cauertun; welce $P$ is ðatte men bugiað Pisse woruld fulneah swilce an Prica for Paet oðer. [Sedgefield, p. 41]

[Alas, Mind, alas; one evil is very much to be avoided. [...] that is the desire for false glory and unrighteous power and unbounded fame of good works among allmen. [...]lt is foolish labor that you struggle all your life to spread your fame without measure over a courtyard such as that in which men inhabit in this world, almost like a point compared with that other. (Traducción propia)] 
[Hélas, Mente, hélas; un mal es de evitarse a toda costa. [...] y es el deseo para la gloria falsa y el poder desviado y la fama sin fin de haber hecho buenas obras entre todos los hombres. [...] Es una labor incorrecta que se lucha toda su vida para ampliar y extender su fama sin medida sobre una corte tal como es la corte que habitan los hombres en este mundo, casi así como un punto a comparación con el otro [mundo]. (Traducción propia )]

A la misma vez, al buscar la fama, igual como en el trabajo para ganar la fama por haber practicado buenas obras, uno descuida las excelencias de la mente. Pero, sor-

prendentemente, Alfred, en otro capítulo de su texto, declara que uno debe seguir el ejemplo de hombres sabios quienes han buscado buena fama (i.e., reputación):

Eala, ge eargan 7 idef geornan; hwy ge swa unnytte sient 7 swa asunde? Hwy ge nyllen ascian after Päm wisum monnǔ 7 aft weorðgeornum, hwylce hi waron Pa Pe aer eow waron? 7 hwy ge Ponne nyllen, siððan ge hiora Peawas geascod habben, him on hirian, swa ge swiðost magen? forðam hi wunnon aft weorðscipe on Pisse worulde, 7 tiolodon goodes hlisan mid goodum weorcǔ, 7 worhton goode bisne Pam Pe after him waron. [Sedgefield, p. 139].

[Alas, you weak and idle! Why are you so useless and languising? Why aren't you willing to seek after wise men and after the men eager for honor? What they were who were before you? And why are you then not willing, after you have found out their manners, to imitate them as best you can? Because they struggled after worship in this world and achieved good reputation for good works and set a good example for those who came after them. (Traducción propia)]

[¡Hélas, que débil y perezoso! ¿Por qué es usted tan inutil y despacio? ¿Por qué no tiene la voluntad de buscar a los hombres sabios y los hombres anxiosos para los honores? [Para saber] lo que eran los que existián antes que usted? Y porque no tiene la voluntad, después que ha entendido sus costumbres, de imitarlos en la mejor manera posible? Porque ellos lucharon para reputación en este mundo y la alcanzaron por sus buenas obras y presentaron un buen ejemplo para los que llegaron después de ellos. (Traducción propia)]

El deseo de gloria mundana, característica a lo que es encomendado culturalmente a un guerrero

anglosajón, persiste aun cuando se está aconsejando humildad y la vida de la mente. En un sentido, 
Alfred está ocupando el antiguo vocabulario anglosajón en que la ansiedad para la fama y gloria y el orgullo sirven para un vocabulario propicio para una exhortación a su pueblo con el propósito de inclinarlos hacia los estudios. No importan las contradicciones: es, mejor dicho, una cuestión de détente entre estudiar para alimentar la mente y la necesidad de defenderse militarmente y avanzar hacia la unificación de la nación.

Mi objetivo no ha sido desenterrar las inconsistencias pequeñas en la obra de Alfred cuando tradujo a Boecio. Ha sido, de otro modo, ilustrar que Alfred encomendó una concepción romana del orden y estabilidad a su pueblo anglosajón en el siglo IX.

Para encomendar esta concepción, Alfred, como rey, líder militar y un líder que encomendó a su pueblo la educación, así como hizo su compatriota Alcuino en el continente europeo, tuvo que traducir esta visión para que sea aceptada por la cultura anglosajón todavía no preparada para asumir la humildad, la renuncia de los bienes de este mundo y la incorporación de la vida de la mente.

A la misma vez, en la traducción cultural de estas ideas-traslatio-de Alfred, perduran las antiguas emociones y concepciones culturales de los Anglosajones. En cuestión de orden, eternidad, fama y el uso de los bienes de este mundo, especialmente para gobernar, Alfred alcanza establecer la superioridad ontológica de un Dios visualizado por su pueblo en el siglo IX como fuerza y sabiduría. Este Dios Alfred representa como la superioridad de la vida de la mente, mientras que tiene que controlar esta tierra desde la eternidad como un jinete guerrero anglosajón-porque no podía negar la experiencia ordinaria de su propia cultura. 
LECTURAS RECOMENDADA:

Alfred. King Alfred's Old English Version of Boethius' De Consolatione Philosophiae

(W.J. Sedgefield, ed. (Oxford, 1899)

Boecio. [Anicius Manlius Torcuatus Severinus Boecio]. The Consolation of

Philosophy. Trans. Victor Watts. (London, 1969)

Boecio. [Anicio Manlio Torcuato Severino Boecio]. La consolación de la filosofía

Trad. Pedro Rodríguez Santidrián. (Madrid, 1999)

Boenig, Robert, ed. Anglo-Saxon Spirituality: Selected Writings (Nueva York, 2000).

Chadwick, Henry. Boecio: the Consolations of Music, Logic, Theology and Philosophy. (Oxford, 1990)

Marenbon, John. Boethius. (New Yorik, 2003)
Mayr-Harting, Henry. The Coming of Christianity to Anglo-Saxon England.

(Pennsylvania, 1991)

Miller Proppe; Katherine. "King Alfred's Consolation of Philosophy" Neuphilolögische MitteiIlungen. (Helsinki, 1973) Tauno Mustanoja, Ed.

Payne, F. Anne. King Alfred and Boecio: an Analysis of the Old English Version Of the Consolation of Philosophy. (Wisconsin, 1968)

Stenton, F. M., Anglo-Saxon England (Oxford, 2001)

Swanton, M.J., (ed. y trad.). The Anglo-Saxon Chronicle (Nueva York, 1998)

Sweet, Henry. Sweet's Anglo-Saxon Dictionary (Oxford, 1897).

Whitelock, Dorothy. Sweet's AngloSaxon reader in Prose and Verse. (Oxford, 2000). 